Kamila Mayumi Duarte Kuabara

\title{
RELAÇÕES CO-FILOGENÉTICAS ENTRE MALÓFAGOS (INSECTA, PHTHIRAPTERA, ISCHNOCERA) E SEUS HOSPEDEIROS GALBULIFORMES
} (AVES)

Co-phylogenetic relationships between chewing lice (Insecta, Phthiraptera, Ischnocera) and their galbuliform hosts (Aves)

Dissertação apresentada ao Programa de Pósgraduação em Sistemática, Taxonomia e Biodiversidade do Museu de Zoologia da Universidade de São Paulo como requisito parcial para obtenção do título de mestre.

Orientador: Dr. Carlos José Einicker Lamas

Coorientador: Dr. Michel Paiva Valim 
"I do not authorize the reproduction and dissemination of this work in part or entirely by any electronic or conventional means."

Catalogação na Publicação

Serviço de Biblioteca e Documentação

Museu de Zoologia da Universidade de São Paulo

Kuabara, Kamila Mayumi Duarte

Relações co-filogenéticas entre malófagos (Insecta, Phthiraptera, Ischnocera) e seus hospedeiros Galbuliformes (Aves) = Co-phylogenetic relationships between chewing lice (Insecta, Phthiraptera, Ischnocera) and their galbuliform hosts (Aves) - Kamila Mayumi Duarte Kuabara; orientador Carlos José Einicker Lamas e coorientador Michel Paiva Valim. São Paulo, 2019.

$62 p$.

Dissertação (Mestrado) - Programa de Pós-Graduação em Sistemática, Taxonomia e Biodiversidade, Museu de Zoologia, Universidade de São Paulo, 2019.

Versão corrigida

1. Ischnocera. 2. Malófagos. I. Lamas, Carlos José Einicker, orient. II. Valim, Michel Paiva, coorient. III.Título.

CDU 595.751

CRB $8 / 3805$ 
"Any worker attempting a natural classification of this difficult group is forced to formulate some conception of its evolution, without which the classification will become even further divorced from a natural representation of relationships than it is at the moment."

Clay (1949, p. 297) 


\section{AGRADECIMENTOS}

O presente trabalho foi financiado em grande parte com o apoio da Fundação de Amparo à Pesquisa do Estado de São Paulo - FAPESP (Processos: 2017/23584-9 e 2017/02014-0), e também à Coordenação de Aperfeiçoamento de Pessoal de Nível Superior - Brasil - CAPES Código de Financiamento 001.

Meus agradecimentos ao Dr. Carlos Lamas pela orientação, apoio e dedicação no desenvolvimento deste projeto e também pelas horas de conversa, incentivo e conselhos sobre a carreira acadêmica e também fora dela.

Agradeço ao Dr. Michel Valim, meu coorientador, amigo e colega de trabalho, que me apresentou e introduziu ao mundo ainda pouco desbravado dos piolhos. Por compartilhar suas ideias, pensamentos e muitas de suas metáforas. Também agradeço pela paciência, dedicação, orientação e acompanhamento durante todos esses anos de trabalho; e por disponibilizar muito do seu tempo para o desenvolvimento deste projeto.

Ao Museu de Zoologia da Universidade de São Paulo (MZUSP), em nome de seus professores e todos os colegas, pelo apoio acadêmico e institucional durante todos os anos em que frequentei o museu durante a minha formação. Pelo auxílio na conferência dos dados das aves, agradeço ao Dr. Luis Fábio Silveira, Msc. Glaucia Del-Rio e à Dr. Natalia Luchetti. Agradeço à Dr. Jaqueline Battilana por todo o auxílio no laboratório de biologia molecular e também as funcionárias da Seção Acadêmica, Marta, Mariana e Sônia, por sempre estarem disponíveis a me receber, esclarecerem dúvidas e agirem em prontidão quando necessitei.

Por ter me recebido de forma tão amável durante meu estágio nos Estados Unidos, e pelas muitas horas conversando sobre piolhos, sou profundamente grata ao Dr. Jason Weckstein. Também agradeço aos Drs. Christopher Witt, Eberhard Mey, Andrew Sweet e Kevin Johnson, por compartilharem seus dados, espécimes e conhecimento em suas respectivas áreas de pesquisa, os quais tiveram grande importância nesse estudo. A Msc. Janice Dispoto por me ajudar em todos os momentos em que precisei, seja no laboratório de biologia molecular ou fora dele, e aos amigos que irão ler isso: Msc. Ana Ferreira, Dr. David Peet, John Hughes, Katie Smith, Moed Gerveni, Dr. Natan Rice, Suravi Ray e Dr. Therese Catanach, obrigada por serem as melhores companhias que eu poderia querer. [For receiving me so friendly during my internship abroad in USA, and for the many hours speaking about louse, my deepest thank you to Dr. Jason Weckstein. To Dr. Christopher Witt, Dr. Eberhard Mey, Dr. Andrew Sweet and Dr. Kevin Johnson, for sharing data, specimens and knowledge of their respective researches which had fundamental meaning on this study, here I express my deepest and sincere thank 
you. I'm very grateful for having Msc. Janice Dispoto to help me in any moment I needed. To the friends who will read this: Msc. Ana Ferreira, Dr. David Peet, John Hughes, Katie Smith, Moed Gerveni, Dr. Natan Rice, Suravi Ray and Dr. Therese Catanach, thank you for being the best companies I could ever asked for].

A minha família, Tiago, mãe, Ju, Sr. Isaac e Mayumi, pois mesmo sem entenderem muito bem a minha pesquisa, nunca deixaram de me apoiar em várias etapas da minha formação profissional e pessoal.

Pelo compartilhamento de ideias e experiências, conversas e risadas agradeço aos meus amigos, Camila Conti, Carol Yamaguchi, Carol Garcia, Cris Apolinário, Fernanda Santos, Isabel Mösch, Jaime Jardim, Marcelo Félix, Marina Lima, Michelle Novais-Vicente, Natan Pedro, Antonio Neto, Renan Carrenho, Rosana Cunha e Virginia Guimarāes.

Caso eu tenha esquecido de alguém, por favor, me desculpem. A todos, minha sincera gratidão. 


\section{RESUMO}

Malófagos desempenham um importante papel na compreensão dos padrões e processos de evolução e em estudos de associação parasito-hospedeiro devido a sua alta especificidade. Foi reconstruída a história filogenética e co-filogenética das espécies dos gêneros Mayriphilopterus (31 espécimes, 9 espécies) e Picicola (38 espécimes, 7 espécies) e seus hospedeiros Galbuliformes (Aves, Bucconidae e Galbulidae), por meio de caracteres moleculares. Foi utilizado um total de 1.464 pares de base, pelo concatenamento do fragmento de um gene mitocondrial COI-r (citocromo oxidase subunidade I - right) e fragmentos de quatro genes nucleares, $E F-1 \alpha$ (fator de elongamento 1 alpha), BR-50, BR-62 e BR-69 para os malófagos e 2.741 pb pelo concatenamento de fragmentos de três genes mitocondriais ND2 (nicotinamida desidrogenase subunidade 2), ND3 (nicotinamida desidrogenase subunidade 3) e cytb (citocromo b) e fragmentos de dois genes nucleares, $A K 1$ (adenilato quinase 1) e BFib7 (beta fibrinogênio 7) para os hospedeiros. Foram feitas análises de máxima verossimilhança, inferência Bayesiana e co-filogenéticas baseada em distância e eventos. As análises filogenéticas recuperaram a monofilia dos hospedeiros e das espécies dos dois gêneros de malófagos. A estatística global da análise co-filogenética de distância foi significativa para os Mayriphilopterus e os Galbuliformes e não significativa para as Picicola. Na análise cofilogenética de eventos recuperou-se para ambos os gêneros, Mayriphilopterus e Picicola, eventos de co-evolução, duplicação e troca de hospedeiro, perdas e falhas em divergir, sendo o último evento o mais comum para os dois gêneros de malófagos. O teste exato de Fisher foi calculado e mostrou que Mayriphilopterus e Picicola não estão co-relacionados entre si, indicando que eles têm histórias evolutivas únicas e independentes em resposta à diversificação dos seus hospedeiros.

Palavras-chave: DNA, JANE, Mayriphilopterus, PARAFIT, Picicola 


\begin{abstract}
Chewing lice play an important role on the understanding of evolutionary patterns in parasitehost studies due to their high specificity. The phylogenetic and cophylogenetic history between Mayriphilopterus (31 specimens, 9 species), Picicola (38 specimens, 7 species) and their galbuliform hosts (Aves, Bucconidae and Galbulidae) was reconstructed, using molecular characters. A total of 1,464 base pairs were used for the concatenation of a fragment of one mitochondrial gene, COI-r (cithocrome oxidase subunit I - right) and fragments of four nuclear genes, $E F-1 \alpha$ (elongation factor 1 alpha), BR-50,BR-62 and BR-69 for chewing lice, and a total of 2,741 bp for the concatenation of three fragments of mitochondrial genes ND2, (NADH dehydrogenase 2), ND3 (NADH dehydrogenase 3) and cytb (cytochrome b) and two fragments of nuclear genes $A K 1$ (adenylate kinase 1) and BFib7 (beta fibrinogen 7) for the hosts. We perfomed Maximum likelihood, Bayesian inference analyzes and the co-phylogenetic analyzes were inferred based on distance and events. The phylogenetic analyzes recovered the monophyly of the hosts and for both Mayriphilopterus and Picicola. The global statistics of cophilogenetic analysis based on distance were significant for Mayriphilopterus and Galbuliformes and not significant for Picicola. For cophylogenetic analysis based on events we recovered events of cospeciation, duplication and host switch, losses and failures to diverge. The failure to diverge was the most common event for both louse genera. Fisher's exact test have showed that Mayriphilopterus and Picicola are not corelated, indicating that they have unique and independent evolutionary histories in response to host diversification.
\end{abstract}

Keywords: DNA, JANE, Mayriphilopterus, PARAFIT, Picicola 


\section{SUMÁRIO}

1. INTRODUÇÃ

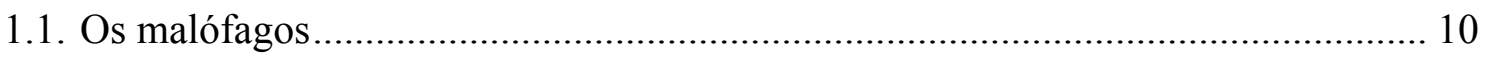

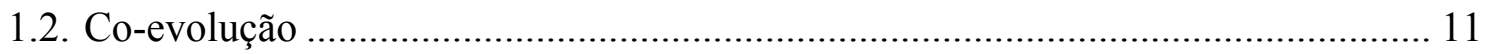

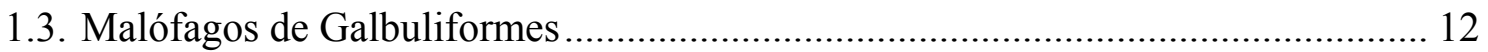

1.3.1. Mayriphilopterus Mey, 2004.............................................................. 12

1.3.2. Picicola Clay et Meinertzhagen, 1938 ........................................................... 12

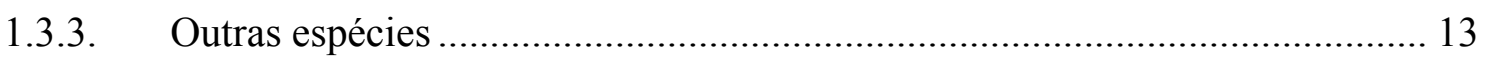

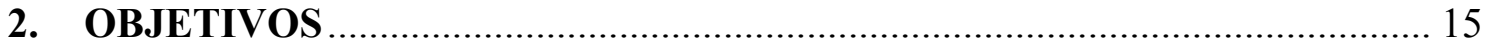

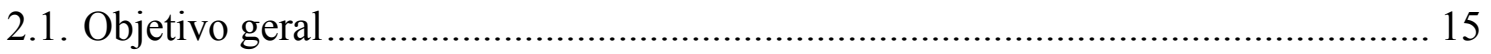

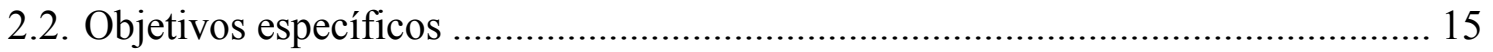

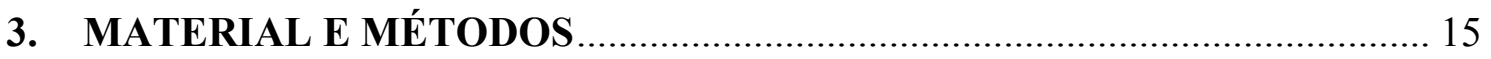

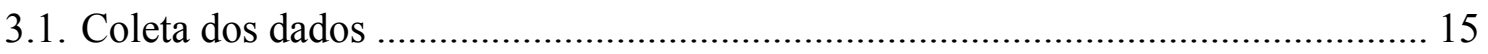

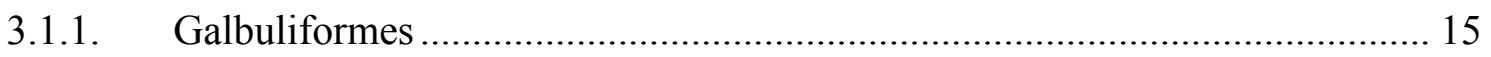

3.1.2. Malófagos ....................................................................................... 16

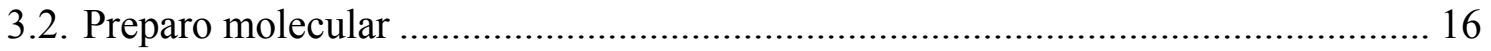

3.2.1. Extração de DNA............................................................................... 16

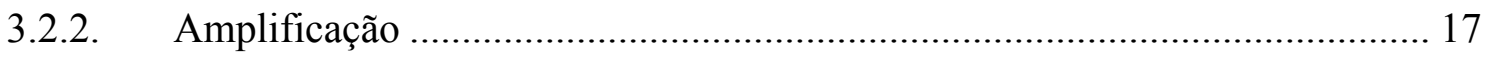

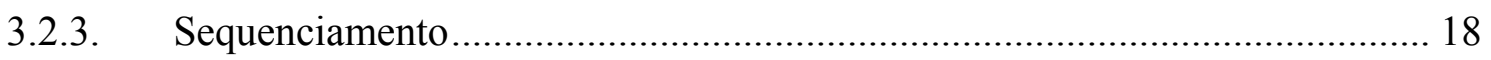

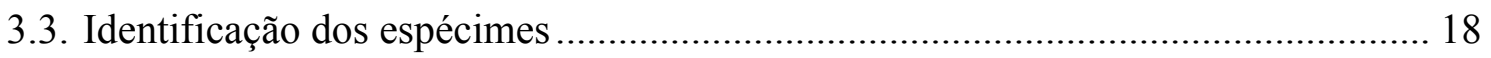

3.3.1. Preparo permanente dos espécimes ............................................................. 18

3.3.2. Delimitação das unidades taxonômicas operacionais (UTOs) ……………..... 18

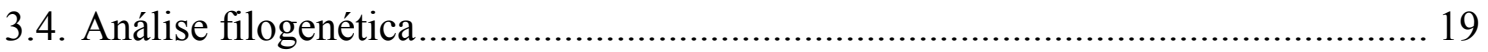

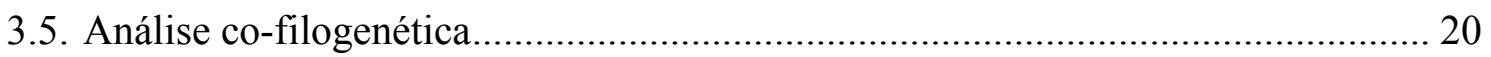

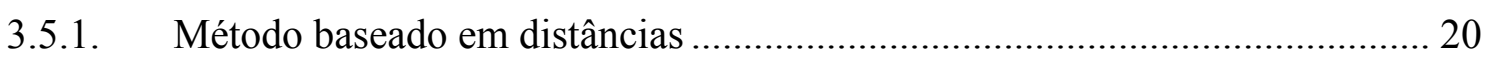

3.5.2. Método baseado em eventos..................................................................... 21

3.5.3. Relações co-filogenéticas compartilhadas...................................................... 21

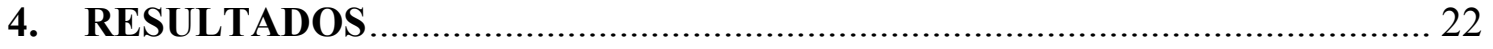

4.1. Identificação dos espécimes ............................................................................ 22

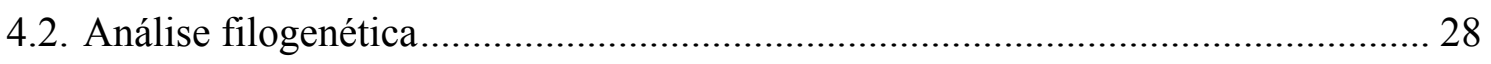

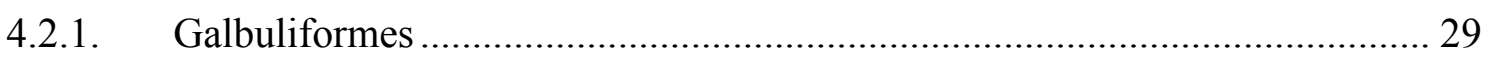

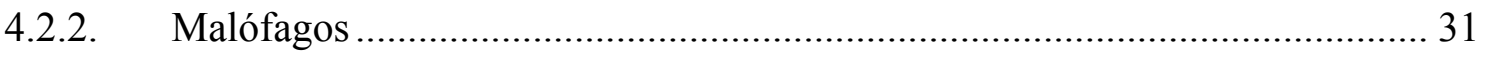

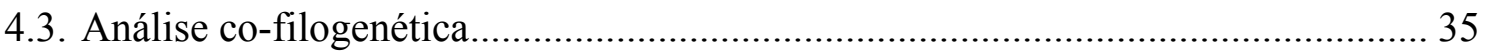

4.3.1. Método baseado em distâncias ...................................................................... 35 


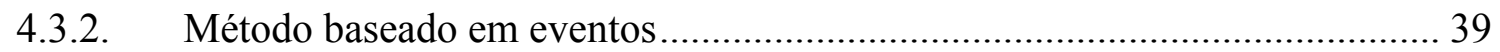

4.3.3. Relações co-filogenéticas compartilhadas ................................................... 43

5. DISCUSSÃ

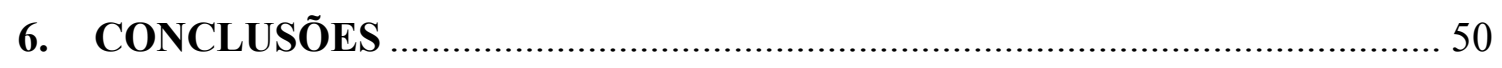

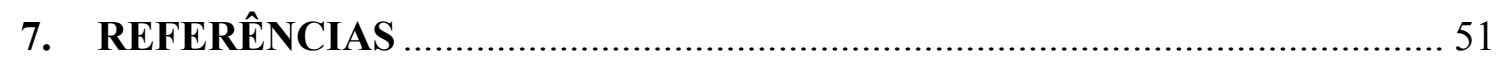

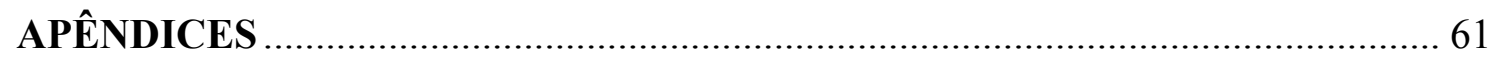




\section{INTRODUÇÃO}

\subsection{Os malófagos}

Os insetos conhecidos popularmente como piolhos, malófagos e anopluros (Insecta, Phthiraptera) são hemimetábolos, incluindo uma fase de ovo, três estágios ninfais e o adulto. São ectoparasitos obrigatórios de aves e mamíferos, vivendo permanentemente todo seu ciclo de vida associados a um único hospedeiro (JOHNSON e CLAYTON, 2003a; LINARDI, 2012). São ápteros, possuem pernas adaptadas para fixação no hospedeiro, com comprimento que varia entre 0,3 e $11 \mathrm{~mm}$, seu corpo é esclerotizado, coberto de cerdas e achatado dorso-ventralmente (JOHNSON e CLAYTON, 2003a).

A ordem Phthiraptera ocorre em todo o mundo e atualmente conta com mais de 5.000 espécies distribuídas em 300 gêneros, estando divididas em quatro subordens: Amblycera, Ischnocera, Rhynchophthirina e Anoplura, sendo as duas últimas encontradas exclusivamente em mamíferos e as duas primeiras as únicas que parasitam aves, e onde encontra-se a maior parte da diversidade do grupo, cerca de 4.000 espécies (PRICE et al., 2003).

Muitos malófagos apresentam alto grau de especificidade, sendo encontrados apenas em grupos (e.g., gênero, família, ordem, etc) de hospedeiros específicos (BECKER, 1933; LAKSHMINARAYANA, 1977; JOHNSON e CLAYTON, 2003b; CLAYTON et al., 2015). Algumas espécies preferem ainda parasitar partes distintas do corpo de seus hospedeiros tais como cabeça, pescoço e/ou asas (CLAY, 1950), apresentando quatro morfotipos específicos desses micro-habitats: piolho de cabeça, piolho de asa, piolho do corpo e generalista (DUBININ, 1938; CLAY, 1949; JOHNSON et al., 2012).

Por viverem todo seu ciclo de vida ligado a um hospedeiro e não conseguirem sobreviver longe destes por muito tempo, pois suas habilidades de dispersão são limitadas, estes insetos são utilizados como um modelo para estudos de co-evolução e de relação parasito-hospedeiro (CLAY, 1949; JOHNSON et al., 2001a). Estes fatores associados a uma ampla distribuição em aves e mamíferos levou muitos autores à conclusão de que os malófagos têm uma longa história de associação e, por consequência, co-evoluem com seus hospedeiros (CLAY, 1949; HOPKINS, 1949; LYAL, 1986; MAUERSBERGER e MEY, 1993; PAGE et al., 1996; HAFNER et al., 2003; BANKS et al., 2006; CLAYTON et al., 2015). 


\subsection{Co-evolução}

Quando duas linhagens interativas estão intimamente associadas durante grande parte ou toda sua diversificação, é possível assumir que a especiação de um grupo seja paralela a do outro. Esse modo de diversificação pode resultar em um padrão de história evolutiva compartilhada entre duas linhagens, conhecida como co-evolução (PATERSON et al., 2003; HUGHES et al., 2007; CLAYTON et al., 2015). Esse processo ocorre em todos os níveis biológicos, como genes atuando dentro de entidades biológicas (e.g. vírus), e mudanças fenotípicas entre duas populações ecologicamente relacionadas, tal como a relação de parasitos e hospedeiros (MARTÍNEZ-AQUINO, 2016).

Os estudos co-evolutivos têm suas origens dentro da parasitologia, pois um dos objetivos dessa disciplina é descrever a "causa e efeito" das interações ecológicas nos sistemas parasito-hospedeiro. A ideia de co-evolução originou-se em estudos parasitológicos de organismos selvagens no início do século XX (FAHRENHOLZ, 1913; KELLOG, 1913; de VIENNE et al., 2013). Esses estudos usavam as relações filogenéticas dos hospedeiros e parasitos e buscavam a congruência filogenética entre eles baseando-se em dois princípios: 1) onde a filogenia do parasito espelha a filogenia de seus hospedeiros, gerando filogenias completamente congruentes (regra de Fahrenholz); e que 2) hospedeiros primitivos albergam parasitos primitivos (regra de Szidat) (FAHRENHOLZ, 1913; SZIDAT, 1940; LYAL, 1986; RÓZSA, 1991; PAGE, 2003). Entretanto, estudos recentes afirmam que filogenias congruentes não são evidências para inferir a co-especiação, mas sim poderosas ferramentas para estudos de co-evolução (PAGE et al., 1998; JOHNSON et al., 2002a; CLAYTON e JOHNSON, 2003; HAFNER et al., 2003; JOHNSON e CLAYTON, 2003b; BANKS e PATERSON, 2004; BANKS et al., 2006; HUGHES et al., 2007; CLAYTON et al., 2015; SWEET et al., 2016; CATANACH et al., 2019).

Os pré-requisitos para a realização de estudos co-filogenéticos, segundo Page et al. (1996), que foram seguidos neste estudo são: (1) ter a taxonomia alpha bem resolvida tanto para os táxons representantes dos parasitos quanto aos táxons representantes dos hospedeiros, (2) ter filogenias bem resolvidas dos parasitos e dos hospedeiros, (3) ter filogenias preferencialmente baseadas em caracteres homólogos (moleculares) entretanto, isso não é um fator limitante, (4) ter um grande número de amostras e, por fim, (5) fazer uma comparação quantitativa das árvores de parasitos e hospedeiros por meio de testes estatísticos. 


\subsection{Malófagos de Galbuliformes}

Os registros de malófagos parasitando as aves da ordem Galbuliformes (Bucconidae e Galbulidae) pertencem a três gêneros da subordem Ischnocera: Mayriphilopterus Mey, 2004, Picicola Clay et Meinertzhagen, 1938 (Apêndice A) e Bobdalgleishia Valim et Cicchino, 2015; e uma única espécie da subordem Amblycera, Menacanthus caudatus (Giebel, 1876).

\subsubsection{Mayriphilopterus Mey, 2004}

O gênero Mayriphilopterus (Ischnocera, Philopteridae) foi descrito por Mey (2004), quando o autor fez a revisão do complexo-Philopterus. Este gênero é encontrado exclusivamente em aves da ordem Galbuliformes. Quatro espécies foram descritas por Mey (2004) e outras duas já haviam sido descritas por Carriker (1963). Após a revisão publicada por Mey (2004) apenas um trabalho foi dedicado ao estudo deste gênero (VALIM e LINARDI, 2007). Atualmente são conhecidas seis espécies de Mayriphilopterus parasitando sete espécies de aves: M. brevicephalus Mey, 2004 ex Jacamerops aureus isidori Deville, 1849; M. ernsti Mey, 2004 ex Monasa morphoeus (Hahn et Küster, 1823); M. galbulicus Mey, 2004 ex Galbula cyanescens Deville, 1849; M. nystalicus Mey, 2004 ex Nystalus chacuru (Vieillot, 1816) e N. maculatus (Gmelin, 1788) (MEY, 2004; VALIM e LINARDI, 2007); M. orinocensis (Carriker, 1963) ex Hypnellus bicinctus Gould, 1837 e M. spinosus (Carriker, 1963) ex Bucco capensis Linnaeus, 1766 (CARRIKER, 1963).

Morfologicamente as espécies de Mayriphilopterus são caracterizadas por serem membros do complexo-Philopterus e apresentarem (1) ausência de conus funcional, (2) trabécula bem desenvolvida, (3) margem hialina do clípeo sem esclerotização marginal e (4) ter de 2-4 pares de cerdas espatuladas na margem hialina do clípeo (MEY, 2004).

\subsubsection{Picicola Clay et Meinertzhagen, 1938}

O gênero Picicola (Ischnocera, Philopteridae) foi descrito por Clay e Meinertzhagen (1938) e atualmente é composto por 37 espécies válidas. Diferente do gênero Mayriphilopterus, o gênero Picicola ocorre em outras ordens de aves, sendo elas Passeriformes (WILLIANS, 1979; CICCHINO, 1981; MEYER et al., 2008), Piciformes (DALGLEISH, 1969) e Galbuliformes (ONIKI e EMERSON, 1981; VALIM e LINARDI, 2006; PRICE e WECKSTEIN, 2006), e há um forte indicativo de que este gênero seja parafilético, com exceção 
das espécies que parasitam as aves da ordem Galbuliformes (WECKSTEIN, 2004a). Atualmente oito espécies de Picicola são conhecidas por parasitar dezessete espécies de Galbuliformes: P. faucetti Price et Weckstein, 2006 ex Chelidoptera tenebrosa (Pallas, 1782); P. galbulica Valim et Linardi, 2006 ex G. cyanescens, G. cyanicollis Cassin, 1851, G. ruficauda Cuvier, 1816 e G. tombacea Spix, 1824; P. naokii Price et Weckstein, 2006 ex B. capensis e B. macrodactylus (Spix, 1824); P. oneilli Price et Weckstein, 2006 ex Notharchus macrorhynchos (Gmelin, 1788); P. osheai Price et Weckstein, 2006 ex Malacoptila fusca (Gmelin, 1788) e $M$. semicincta Todd, 1925; P. serrafreirei Valim et Linardi, 2006 ex N. chacuru e N. maculatus; P. striata Oniki et Emerson, 1981 ex Malacoptila striata (Spix, 1824), M. fulvogularis Sclater, 1854, Hapaloptila castanea (Verreaux, 1866) e Monasa nigrifrons (Spix, 1824) e P. valquii Price et Weckstein, 2006 ex Nothriscus tectus (Boddaert, 1783) (ONIKI e EMERSON, 1981; PRICE et al., 2003; VALIM e LINARDI, 2006; PRICE e WECKSTEIN, 2006).

Morfologicamente as espécies de Picicola são caracterizadas por serem membros do complexo-Degeeriella e por apresentarem (1) margem anterior da cabeça de forma arredondada ou achatada, (2) carena marginal completa, (3) sutura pré-antenal completa e (4) cônus estreito e alongado (ONIKI e EMERSON, 1981; VALIM e LINARDI, 2006; PRICE e WECKSTEIN, 2006).

\subsubsection{Outras espécies}

O gênero monotípico Bobdalgleishia stephanophallus (Ischnocera, Philopteridae) foi descrito por Valim e Cicchino (2015), e pertence ao complexo-Brueelia. Esta é a única espécie deste complexo conhecida por parasitar aves da ordem Galbuliformes. Seu hospedeiro tipo é Jacamerops aureus ridgwayi Todd, 1943 (Galbulidae). Morfologicamente essa espécie é caracterizada por apresentar (1) as cerdas $o s, m t s 1-3$ longas na margem da têmpora, (2) cerda pos curta e alocada na lente ocular, (3) um par de cerdas na porção anterior do tergito II e (4) esternitos II-VI com mais de um par de cerdas e sem placas esclerotizadas.

A espécie Menacanthus caudatus (Amblycera, Menoponidae) foi descrita por Giebel (1876), como Menopon caudatus em Galbula ruficauda, e é membro do complexoMenacanthus Neumann, 1912. Esta é a única espécie de Amblycera conhecida por parasitar Galbuliformes. A checklist de Hopkins e Clay (1952) e a revisão do gênero Menacanthus de Piciformes de Price e Emerson (1975) classificam esta espécie como nomina dubia por apresentar grande dúvida quanto a sua validade morfológica uma vez que o material tipo está 
perdido, a descrição original é incompleta e a falta de recoleta dessa espécie em G. ruficauda na natureza.

Devido à falta de material disponível para extração de DNA de $B$. stephanophallus e $M$. caudatus, essas espécies não foram incluídas neste trabalho. 


\section{CONCLUSÕES}

Foi recuperada a monofilia de todas as espécies de malófagos do gênero Mayriphilopterus.

Assim como os Mayriphilopterus, as espécies do gênero Picicola que parasitam aves da ordem Galbuliformes se mostraram monofiléticas.

As aves das famílias Bucconidae e Galbulidae, hospedeiros das espécies dos gêneros Mayriphilopterus e Picicola, apresentaram-se monofiléticas.

Foram recuperados mais eventos de co-especiação entre os Mayriphilopterus e seus hospedeiros do que entre as Picicola e os Galbuliformes, e a significância estatística foi positiva apenas entre os Mayriphilopterus e os Galbuliformes.

Baseado nos dois tipos de análises co-filogenéticas (eventos e distâncias), nenhum dos dois gêneros de malófagos apresentaram padrões perfeitos de co-evolução com seus hospedeiros. Fatores ecológicos (e.g. foresia e preening) podem estar delimitando os padrões de parasitismo observados. 


\section{REFERÊNCIAS}

Altschul, S.F.; Gish, W.; Miller, W.; Myers, E.W.; Lipman, D.J. 1990. Basic local alignment search tool. Journal of Molecular Biology, 215(3): 403-10. doi: 10.1016/S00222836(05)80360-2

Arzua, M.; Valim, M.P. 2010. Bases para o estudo qualitativo e quantitativo de ectoparasitos de aves. In: Von Matter, S.; Straube, F.C.; Accordi, I.A.; Piacentini, V.Q.; Cândido Jr., J.F. (Eds.). Ornitologia e Conservação: Ciência Aplicada, Técnicas de Pesquisa e Levantamento. Rio de Janeiro, Technical Books, Cap. 15, p. 1-17.

Banks, J.C.; Paterson, A.M. 2004. A penguin-chewing louse (Insecta: Phthiraptera) phylogeny derived from morphology. Invertebrate Systematics, 18: 89-100. doi: 10.1071/IS03022

Banks, J.C.; Palma, R.L.; Paterson, A.M. 2006. Cophylogenetic relationships between penguins and their chewing lice. Journal of Evolutionary Biology, 19: 156-166. doi: 10.1111/j.1420-9101.2005.00983.x

Bartlow, A.W.; Villa, S.M.; Thompson, M.W.; Bush, S.E. 2016. Walk or ride? Phoretic behaviour of amblyceran and ischnoceran lice. International Journal for Parasitology, 46(4): 221-227. doi: 10.1016/j.ijpara.2016.01.003

Becker, E.R. 1933. Host-specificity and specifity of the animal parasites. American Journal of Tropical Medicine, 13: 505-523.

Benjamini, Y.; Hochberg, Y. 1995. Controlling the false discovery rate: a practical and powerful approach to multiple testing. Journal of the Royal Statistical Society, 57: 289300. doi: $10.2307 / 2346101$

Bush, S.E.; Dukgun, K.; Reed, M.; Clayton, D.H. 2010. Evolution of cryptic coloration in Ectoparasites. The American Naturalist, 176(4): 529-535. doi: 10.1086/656269

Carriker Jr., M.A. 1963. New and little known Mallophaga from Venezuelan birds (Part II). Memoria Sociedad de Ciencias Naturales La Salle, 23(64): 5-42.

Catanach, T.A.; Valim, M.P.; Weckstein, J.D.; Johnson, K.P. 2017. Cophylogenetic analysis of lice in the Colpocephalum complex (Phthiraptera: Amblycera). Zoologica Scripta, 47: 72 83. doi: $10.1111 /$ zsc. 12262

Catanach, T.A.; Johnson, K.P.; Marks, B.D.; Moyle, R.G.; Valim. M.P.; Weckstein, J.D. 2019. Two lineages of kingfisher feather lice exhibit different degrees of cospeciation with their hosts. Parasitology, 1-13. doi: 10.1017/S0031182019000453 
Cicchino, A.C. 1981. Contribución al conocimiento de los malófagos argentinos XI. Dos nuevas especies del subgénero Picicola (Tyrannicola) Carriker, 1956, parasitas de Furnariidae (Aves: Passeriformes). Revista de la Sociedad Entomologica Argentina, 40: $279-283$.

Clay, T.; Meinertzhagen, R. 1938. Two new genera of Mallophaga. The Entomologist, 71(899): 73-76.

Clay, T.; Meinertzhagen, R. 1943. The relationship between Mallophaga and hippoboscid flies. Parasitology, 35(1-2): 11-16.

Clay, T. 1949. Some problems in the evolution of a group of ectoparasites. Evolution, 3: 279299.

Clay, T. 1950. A preliminary survey of the distribution of the Mallophaga ("feather lice") on the class Aves (birds). Journal of the Bombay Natural History Society, 49(3): 430-443.

Clay, T. 1951. An introduction to a classification of the avian Ischnocera (Mallophaga): Part I. Transactions of the Royal Entomological Society of London, 102: 171-195.

Clayton, D.H. 1990. Host specificity of Strigiphilus Owl lice (Ischnocera: Philopteridae), with the description of new species and host associations. Journal of Medical Entomology, 27(3): 257-265. doi:10.1093/jmedent/27.3.257

Clayton, D.H.; Price, R.D.; Page, R.D.M. 1996. Revision of Dennyus (Collodennyus) lice (Phthiraptera: Menoponidae) from swiftlets, with descriptions of new taxa and a comparison of host-parasite relationships. Systematic Entomology, 21: 179-204. doi: 10.1046/j.1365-3113.1996.d01-13.x

Clayton, D.H.; Walther, B.A. 1997. Collection and quantification of arthropod parasites of birds. In Clayton, D.H.; Moore, J. (Eds.). Host-parasite evolution: general principles and avian models. Oxford, Oxford University Press. p. 419-440.

Clayton, D.H.; Johnson, K.P. 2003. Linking coevolutionary history to ecological process: Doves and lice. Evolution, 57(10): 2335-2341.

Clayton, D.H.; Al-Tamimi, S.; Johnson, K.P. 2003. The ecological basis of coevolutionary history. In: Page, R.D.M. (Ed.). Tangled trees: phylogeny, cospeciation and coevolution. Chicago, The University of Chicago Press. p.195-220.

Clayton, D.H.; Bush, S.E.; Johnson, K.P. 2015. Coevolution of life on hosts. Chicago, The University of Chicago Press. 320p. 
Clement, M.; Snell, Q.; Wwalke, P.; Posada, D.; Crandall, K. 2002. TCS: estimating gene genealogies. In: International Parallel Distributed Processing Symposium, $16^{\circ}$. Proceeding IEEE Computing Society. p. 184.

Conow, C.; Fielder, D.; Ovadia, Y.; Libeskind-Hadas, R. 2010. Jane: a new tool for the cophylogeny reconstruction problem. Algorithms for molecular biology, 5(10): 1-10. doi: 10.1186/1748-7188-5-16

Dalgleish, R.C. 1969. The Picicola (Phthiraptera: Ischnocera) of the Picidae (Aves: Piciformes). Proceedings of the Royal Entomological Society of London (Series B), 38: $101-113$.

Danfort, B.N.; Ji, S. 1998. Elongation factor-1 $\alpha$ occurs as two copies in bees: implications for phylogenetic analysis of EF-1 $\alpha$ sequences in insects. Molecular biology and Evolution, 15: 225-235.

de Moya, R.; Allen, J.M.; Sweet, A.D.; Walden, K.K.O.; Palma, R.L.; Smith, V.S..; Cameron, S.L.; Valim, M.P.; Galloway, T.D.; Weckstein, J.D.; Johnson, K.P. In prep. The erlist codivergence in avian feather lice indicates extensive host-switching after the Cretaceous-Paleogene mass extinction event.

de Vienne, D.M.; Refrégier, G.; López-Villavicencio, M.; Tellier, A.; Hood, M.E.; Giraud, T. 2013. Cospeciation vs host-shift speciation: methods for testing, evidence from natural associations and relation to coevolution. New Phytologist, 198(2): 347-385. doi: $10.1111 / \mathrm{nph} .12150$

Doyon, J.P.; Ranwez, V.; Daubin, V.; Berry, V. 2011. Models, algorithms and programs for phylogeny reconciliation. Briefings in Bioinformatics, 12(5): 392-400. doi: 10.1093/bib/bbr045

Dubinin, D.B. 1938. Ismenenija parasitofauny karavajki (Plegadis falcinellus) vysyvajemyje vosrostorn i migracjej chosiaina. Trudy Astrachanskogo Gosudarstvennogo Zapovednika, 2: 114-212.

Escalante, G.C.; Sweet, A.D.; Mccracken, K.G.; Gustafsson, D.R.; Wilson, R.E.; Johnson, K.P. 2016. Patterns of cryptic host specificity in duck lice based on molecular data. Medical and Veterinary Entomology, 30(2): 200-208. doi: 10.1111/mve.12157

Fahrenholz, H. 1913. Ectoparasiten und abstammungslehre. Zoologischer Anzeiger, 41: 371374. 
Ferreira, M. 2018. Filogenia e biogeografia de três famílias de aves do Neotrópico. 115 f. Tese (Doutorado em Genética, Conservação e Biologia Evolutiva) - Instituto Nacional de Pesquisas da Amazônia, Amazonas.

Filipiak, A.; Zając, K.; Kübler, D.; Kramarz, P. 2016. Coevolution of host-parasite associations and methods for studying their cophylogeny. Invertebrate Survival Journal, 13: 56-65.

Fisher, R.A. 1925. Statistical methods for research workers. Edimburgh, Oliver \& Boyd. $362 \mathrm{p}$.

Folmer, O.; Black, M.; Hoew, W.; Lutz, R.; Vrijenhoek, R. 1994. DNA primers for amplification of mithocondrial cytochrome c oxidase subunit I from diverse metazoan invertebrates. Molecular Marine Biology and Biotechnology, 3(5): 294-299.

Giebel, C.G.A. 1876. Zwei neue Epizoen (Nirmus sulphureus and Menopon caudatum) von Trogon und Galbula ruficauda. Zeitschrift für die gesammten Naturwissenschaften, 47: $528-529$.

Hafner, M.S.; Sudman, P.D.; Villablance, F.X.; Spradling, T.A.; Demastes J.W.; Nadler, S.A. 1994. Disparate rates of molecular evolution in cospeciating hosts and parasites. Science, 365: $1087-1090$.

Hafner, M.S.; Demastes, J.W.; Spradling, T.A.; Reed, D.L. 2003. Cophylogeny between pocket gophers and chewing lice. In: Page, R.D.M. (Ed.). Tangled trees: phylogeny, cospeciation and coevolution. Chicago, The University of Chicago Press. p.195-220.

Hammer, S.; Brown, R.; Bugoni, L.; Palma, R.; Hughes, J. 2010. On the origin of Halipeurus heraldicus on Round Island petrels: Cophylogenetic relationships between petrels and their chewing lice. Molecular Phylogenetics and Evolution, 55: 1111-1120. doi:10.1016/j.ympev.2010.01.013

Harbison, C.W.; Bush, S.E.; Malenke, J.R.; Clayton, D.H. 2008. Comparative transmission dynamics of competing parasites species. Ecology, 89(11): 3186-3194. https://doi.org/10.1890/07-1745.1

Hopkins, G.H.E. 1949. The host associations of the lice of mammals. Proceedings of the Zoological Society of London, 119: 387-604.

Hopkins, G.H.E.; Clay, T. 1952. A check list of the genera \& species of Mallophaga. London, British Museum of Natural History. 362p.

Hughes, J.; Kennedy, M.; Johnson, K.P.; Palma, R.L.; Page, R.D.M. 2007. Multiple cophylogenetic analysis reveals frequent cospeciation between Pelicaniform birds and Pectinopygus lice. Systematic Biology, 56(2): 232-251. 
Huson, D.H.; Scornavacca, C. 2012. Dendroscope 3: An interactive tool for rooted phylogenetic trees and network. Systematic Biology, 61(6): 1061-1067. doi: 10.1093/sysbio/sys062

Johnson, K.P.; Drown, D.M.; Clayton, D.H. 2001a. A data based parsimony method of cophylogenetic analysis. Zoologica Scripta, 30(2): 79-87. doi: 10.1046/j.14636409.2001.00057.x

Johnson, K.P.; Adams, R.J.; Clayton, D.H. 2001b. Molecular systematics of Goniodidae (Insecta: Phthiraptera). Journal of Parasitology, 87(4): 862-869.

Johnson, K.P.; Adams, R.J.; Clayton, D.H. 2002a. The phylogeny of the louse genus Brueelia does not reflect host phylogeny. Biological Journal of the Linnean Society, 77: 233-247. doi: 10.1046/j.1095-8312.2002.00107.x

Johnson, K.P.; Weckstein, J.D.; Witt, C.C.; Faucett, R.C.; Moyle, R.G. 2002b. The perils of using host relationships in parasite taxonomy: Phylogeny of the Degeeriella complex. Molecular Phylogenetics and Evolution, 23: 150-157.

Johnson, K.P.; Williams, B.L.; Drown, D.M.; Adams, R.J.; Clayton, D.H.. 2002c. The population genetics of host specificity: genetic differentiation in dove lice (Insecta: Phthiraptera). Molecular Ecology, 11: 25-38.

Johnson, K.P.; Clayton, D.H. 2003a. The biology, ecology, and evolution of chewing lice. In: Price, R.D.; Hellental, R.A.; Palma, R.L.; Johnson, K.P.; Clayton, D.H. (Eds.). The chewing lice: world checklist and biological overview. Champaign, Natural History Survey. p. 449-476. (Special Publication)

Johnson, K.P.; Clayton, D.H. 2003b. Coevolutionary history of ecological replicates: comparing phylogenies of wing and body lice to Columbiform hosts. In: Page, R.D.M. (Ed.). Tangled trees: phylogeny, cospeciation and coevolution. Chicago, The University of Chicago Press. p. 262-286.

Johnson, K.P.; Shreve, S.M.; Smith, V.S. 2012. Repeated adaptive divergence of microhabitat specialization in avian feather lice. BMC Biology, 10(52): 1-11. doi: 10.1186/1741-7007$10-52$

Kearse, M.; Moir, R.; Wilson, A.; Stones-Havas, S.; Cheung, M.; Sturrock, S.; Buxton, S.; Cooper, A.; Markowitz, S.; Duran, C. 2012. Geneious basic: an integrated and extendable desktop software platform for the organization and analysis of sequence data. Bioinformatics, 28: 1647-1649. doi: 10.1093/bioinformatics/bts199

Keirans, J.E. 1975. A review of the phoretic relationship between Mallophaga (Phthiraptera: Insecta) and Hippoboscidae (Diptera: Insecta). Journal of Medical Entomology, 12(1): 
$71-76$.

Kellogg, V.L. 1913. Distribution and species-forming of ecto-parasites. The American Naturalist, 47:129-158.

Kumar, S.; Stecher, G.; Tamura, K. 2016. MEGA7: Molecular Evolutionary Genetics Analysis version 7.0 for bigger datasets. Molecular Biology and Evolution, 33: 1870-1874. doi: 10.1093/molbev/msw054

Lakshminarayana, K.V. 1977. Factors involved in the host-specificity in Mallophaga sens. lat., (Phthiraptera: Insecta) infesting birds. In: Ananthakrishnan, T.N. (Ed.). Insects and hostspecificity: Proceedings of the Symposium on Problems of Host-specificity in Insects. Madras, The Macmillan Company of India. p. 101-109.

Lanfear, R.; Frandsen, P.B.; Wright, A.M.; Senfeld, T.; Calcott, B. 2016. PartitionFinder2: new methods for selecting partitioned models of evolution for molecular and morphological phylogenetic analyses. Molecular biology and evolution, 34(3): 772-773. doi: 10.1093/molbev/msw260

Legendre, P.; Desdevises, Y.; Bazin, E. 2002. A statistical test for host-parasite coevolution. Systematic Biology, 51: 217-234.

Leigh, J.W.; Bryant, D. 2015. Popart: full-feature software for haplotype network construction. Methods in Ecology and Evolution, 6(9): 1110-1116. doi: 10.1111/2041-210X.12410 Linardi, P.M. 2012. Phthiraptera. In: Rafael, J.A.; Melo, G.A.R.; Carvalho, C.J.B.; Casari, S.A.; Constantino, R. (Eds.). Insetos do Brasil: diversidade e taxonomia. Ribeirão Preto, Ed. Holos. cap. 31, p. 439-451.

Lyal, C.H.C. 1986. Coevolutionary relationships of lice and their hosts: a test of Farenholz's Rule. In: Stone, A.R.; Hawksworth, D.L. (Eds.). Coevolution and Systematics. Oxford, Ed. Claredon Press, p. 77-91.

Malenke, J.R.; Johnson, K.P.; Clayton, D.H. 2009. Host specialization differentiates cryptic species of feather-feeding lice. Evolution, 63(6): 1427-1438. doi: 10.1111/j.15585646.2009.00642.x.

Martínez-Aquino, A. 2016. Phylogenetic framework for coevolutionary studies: a compass for exploring jungles of tangled trees. Current Zoology, 62(4): 393-403. doi: 10.1093/cz/zow018

Mauersberger, G.; Mey, E. 1993. Mallophagen und Vogelsystem - Beitrag zur Diskussion der Parasitophyletik. Mitteilungen aus dem Zoologischen Museum in Berlin, 69(Suppl.): 330. 
Merilä, J.; Wiggins, D. 1995. Interspecific competition for nest holes causes adult mortality in the Collared Flycatcher. Condor, 97(2): 445- 450.

Mey, E. 1994. Beziehungen zwischen Larvenmorphologie und Systematik der Adulti bei den Vogel-Ischnozeren (Insecta, Phthiraptera, Ischnocera). Mitteilungen aus dem Zoologischen Museum in Berlin, 70(1): 3-84.

Mey, E. 2004. Zur taxonomie, verbreitung und parasitophyletischer evidenz des PhilopterusKomplexes (Insecta, Phthiraptera, Ischnocera). Ornithologischer Anzeiger, 43: 149-203. Meyer, M.J.; Price, R.D.; Johnson, K.P. 2008. A new species of Picicola Clay and Meinertzhagen, 1938 (Phthiraptera: Ischnocera) parasitic on the Rufous-sided Broadbill (Passeriformes: Eurylaimidae) in Ghana. Zootaxa, 1762: 63-68. doi: 10.5281/zenodo.181951

Miller, M.A.; Pfeiffer, W.; Schwartz T. 2010. Creating the CIPRES Science Gateway for inference of large phylogenetic trees. In: Proceedings Gateway Computing Enviroments Workshop (GCE). New Orleans. p. 1-8.

Nordberg, S. 1936. Biologisch-ökologische Untersuchungenüber die Vogelnidocolen. Acta Zoologica Fennica, 21: 1-168.

Oniki, Y.; Emerson, K.C. 1981. A new species of Picicola (Mallophaga: Philopteridae) from the crescent-chested puffbird Malacoptila striata (Spix) (Piciformes, Bucconidae). Revista Brasileira de Biologia, 41(3): 511-513.

Page, R.D.M. 2003. Tangled trees: Phylogeny, Cospeciation and Coevolution. Chicago, The University of Chicago Press, 350 p. doi: 10.1111/j.1439-0310.2004.00990.x

Page, R.D.M.; Clayton, D.H.; Paterson, A.M. 1996. Lice and cospeciation: a response to Barker. International Journal for Parasitology, 26(2): 213-218.

Page, R.D.M.; Lee, P.M.L.; Becher, S.A.; Griffiths, R.; Clayton, D.H. 1998. A different tempo of mitochondrial evolution in birds and their parasitic lice. Molecular Phylogenetics and Evolution, 9(2): 276-293.

Palma, R.L. 1978. Slide-mounting of lice: a detailed description of the Canada balsam technique. New Zealand Entomologist, 6(4): 432-436.

Paradis, E.; Claude, J.; Strimmer, K., 2004. APE: analyses of phylogenetics and evolution in R language. Bioinformatics, 20. doi: 10.1093/ bioinformatics/btg412.

Paterson, A.M.; Palma, R.L.; Gray, R.D. 2003. Drowning on arrival, missing the boat and xevents: How likely are sorting events? In: Page, R.D.M. (Ed.). Tangled Trees phylogeny, cospeciation and coevolution. Chicago, The University of Chicago Press. p. 287-309. 
Price, R.D.; Hellenthal, R.A.; Palma, R.L. 2003. World checklist of chewing lice with host associations and keys to families and genera. In: Price, R.D.; Hellental, R.A.; Palma, R.L.; Johnson, K.P.; Clayton, D.H. (Eds.). The chewing lice: world checklist and biological overview. Champaign, Illinois Natural History Survey. p. 1-448. (Special Publication)

Price, R.D.; Emerson, K.C. 1975. The Menacanthus (Mallophaga: Menoponidae) of the Piciformes (Aves). Annals of the Entomological Society of America, 68(5): 779-785.

Price, R.D.; Weckstein, J.D. 2006. Picicola Clay \& Meinertzhagen (Phthiraptera: Philopteridae) from jacamars and puffbirds (Piciformes: Galbulidae, Bucconidae), with descriptions of five new species. Zootaxa, 1367: 37-50. doi: 10.5281/zenodo.174824

Prum, R.O.; Berv, J.S.; Dornburg, A.; Field, D.J.; Townsend, J.P.; Lemmon, E.M.; Lemmon, A.R. 2015. A comprehensive phylogeny of birds (Aves) using targeted next-generation DNA sequencing. Nature, 526: 569-573. doi:10.1038/nature15697

Puillandre, N.; Lambert, A.; Brouillet, S.; Achaz, G. 2012. ABGD, Automatic Barcode Gap Discovery for primary species delimitation. Molecular Ecology, 21(6): 1864-1877. doi: $10.1111 / \mathrm{j} .1365-294 X .2011 .05239$

R Core Team. 2008. R: A language and environment for statistical computing. Disponível em: http://www.R-project.org.

Rambaut, A. 2007. FigTree v1.4.0, a graphical viewer of phylogenetic trees. Disponível em: http://bio.ed.ac.uk/software/figtree/.

Rambaut, A.; Drummond, A.J. 2009. Tracer v.1.5. Disponível em: https://github.com/evolvedmicrobe/beast-mcmc/tree/master/release_tracer/Windows /Tracer\%20v1.5

Ronquist, F.; Teslenko, M.; Van Der Mark, P.; Ayres, D. L.; Darling, A.; Hohna, S. 2012. MrBayes 3.2: Efficient Bayesian Phylogenetic Inference and model choice across a large model space. Systematic Biology, 61(3): 539-542. doi: 10.1093/sysbio/sys029

Rózsa, L. 1991. Flamingo lice contravene Fahrenholz. International Journal for Parasitology, 21(2): 151-152.

Sick, H. 2001. Ornitologia Brasileira. Rio de Janeiro, Nova Fronteira. 912p.

Stamatakis, A. 2014. RAxML version 8: A tool for phylogenetic analysis and post- analysis of large phylogenies. Bioinformatics, 30: 1312-1313. doi: 10.1093/bioinformatics/btu033

Sugiura, N. 1978. Further analysis of the data by akaike' s information criterion and the finite corrections. Communications in Statistics - Theory and Methods, 7(1): 13-26. doi: $10.1080 / 03610927808827599$ 
Sweet, A.D.; Allen, J.M.; Johnson, K.P. 2014. Novel Primers from informative nuclear loci for louse molecular phylogenetics (Insecta: Phthiraptera). Journal of Medical Entomology, 51(6): 1122-26. doi: 10.1603/ME13218.

Sweet, A.D.; Boyd, B.M.; Johnson, K.P. 2016. Cophylogenetic patterns are uncorrelated between two lineages of parasites on the same hosts. Biological Journal of the Linnean Society, 118: 813-828. doi: 10.1111/bij.12771

Sweet, A.D.; Boyd, B.M.; Allen, J.M.; Villa, S.M.; Valim, M.P.; Rivera-Parra, J.L.; Wilson, R.E.; Johnson, K.P. 2017. Integrating phylogenomic and population genomic patterns in avian lice provides a more complete picture of parasite evolution. Evolution, 72(1): 95112. doi: 10.1111/evo.13386

Szidat, L. 1940. Beiträge zum Aubfau eines natürlichen Systems der Trematoden. I. Die Entwicklung von Echinocercaria choanophila U. Szidat zu Cathaemasia hians und die Ableitung der Fasciolidae von den Echinostomidae. Zeitschrift für Parasitenkunde, 11: $239-283$.

Valim, M.P.; Linardi, M.P. 2006. Two new species of Picicola Clay \& Meinertzhagen, 1938 (Phthiraptera: Philopteridae) from Piciformes (Bucconidae and Galbulidae) in Brazil. Zootaxa, 1172: 21-29. doi: 10.11646/zootaxa.1172.1.2

Valim, M.P.; Linardi, M.P. 2007. Description of the male of Mayriphilopterus nystalicus Mey, 2004 (Phthiraptera, Ischnocera, Philopteridae). Zootaxa, 1604: 47-51. doi: 10.11646/zootaxa.1604.1.4

Valim, M.P.; Weckstein, J.D. 2011. Two new species of Brueelia Kéler, 1936 (Ischnocera, Philopteridae) parasitic on Neotropical trogons (Aves, Trogoniformes). Zookeys, 128: 113. doi: 10.3897/zookeys.128.1583

Valim, M.P.; Weckstein, J.D. 2012. Two new species of Cotingacola Carriker, 1956 (Phthiraptera: Ischnocera: Philopteridae) from Amazonian Brazil, with comments on hostspecificity. Systematic Parasitology, 81: 159-167. doi 10.1007/s11230-011-9334-7

Valim, M.P.; Cicchino, A.C. 2015. A remarkable new genus and a new species of chewing louse (Phthiraptera, Ischnocera, Philopteridae) from Brazil. Zookeys, 541: 57-70. doi: 10.3897/zookeys.541.6022

Weckstein, J.D. 2004a. Systematics and cophylogenetics of toucans and their associated chewing lice. 139 f. Tese (Doutorado em Filosofia) - Louisiana State University, Louisiana. Weckstein, J.D. 2004b. Biogeography explains cophylogenetic patterns in toucan chewing lice. Systematic Biology, 53: 154-164. doi: 10.1080/10635150490265085 
Willians, N.S. 1979. The Picicola (Mallophaga: Philopteridae) of the Passeriformes (Aves). Journal of Kansas Entomological Society, 52(4): 633-640.

Witt, C.C. 2004. Rates of molecular evolution and their application to Neotropical avian biogeography. 138 f. Tese (Doutorado em Filosofia) - Louisiana State University, Louisiana. 